\title{
CLASSIFICAÇÃO DE USO DO SOLO URBANO ATRAVÉS DA ANÁLISE LINEAR DE MISTURA ESPECTRAL COM IMAGENS DE SATÉLITE*
}

\author{
JORGE ROCHA ${ }^{1}$ \\ JosÉ ANTÓNIO TENEdÓRIOº \\ ROSSANA ESTANQUEIRO ${ }^{2}$ \\ Paulo Morgado Sousa ${ }^{1}$
}

\begin{abstract}
Resumo - Os ambientes urbanos são heterogéneos, sendo útil simplificá-los em combinações de materiais básicos de uso e ocupação do solo a fim de permitir estudos quantitativos. O modelo VIS proposto por Ridd em 1995 é um modelo conceptual que permite simplificar ambientes urbanos através da combinação de três componentes básicos: vegetação $(V)$, superfície impermeável $(I)$ e solo $(S)$. A maioria dos usos urbanos pode ser interpretada à luz de combinações destes três componentes básicos. A análise da composição do VIS permite revelar que a maioria das características urbanas tem a sua própria composição original destes três factores, a qual é difícil de observar através dos métodos de classificação de imagens de satélite que operam ao nível do pixel. Este trabalho examina as características das classes de uso e ocupação do solo urbano na Grande Área Metropolitana de Lisboa (GAML) utilizando técnicas de classificação baseadas no sub-pixel, nomeadamente a Análise de Mistura Espectral (AME), desenvolvendo um modelo conceptual para caracterizar os padrões de ocupação, sendo explorada e testada a aplicabilidade da AME para medir a composição física da morfologia urbana, através de imagens multiespectrais Landsat 7 ETM+ e SPOT 5 HRVIR, avaliando, ao mesmo tempo, o efeito que as diferenças de resolução espacial podem ter nos resultados obtidos.
\end{abstract}

Palavras-chave: Detecção remota, Análise de Mistura Espectral, uso do solo urbano.

\begin{abstract}
URban LAND USE CLASSIFICATION THROUGH SATELLITE IMAGES SPECTRAL MIXTURE LINEAR ANALYSIS. Urban environments are heterogeneous by nature. Hence, to allow quantitative studies it's necessary to simplify them in combinations of basic land use/cover materials. The Ridd's VIS model (1995) is a conceptual representation that allows simplifying urban environments through the combination of three basic components: vegetation (V), impervious surface (I) and soil (S). The majority of urban uses can be interpreted as a combination of those three basic components.
\end{abstract}

* Recebido: 25/01/2006. Revisto: 10/07/2007. Aceite: 24/07/2007.

1 Investigadores do Centro de Estudos Geográficos, Universidade de Lisboa. E-mail: jrocha@fl.ul.pt e pms@fl.ul.pt

2 Investigadores do e-GEO, Universidade Nova de Lisboa. E-mail: ja.tenedorio@fcsh.unl.pt e rossana.estanqueiro@fcsh.unl.pt 
The VIS analysis allows to disclose that the mainstream of urban features has it's own VIS signature, which is difficult to detect through pixel-by-pixel based classifiers. This work examines the land use/cover characteristics of the Great Lisbon Metropolitan Area (GAML) using sub-pixel classification techniques, mainly linear spectral unmixing (LSU), developing a conceptual model to characterize the occupation standards. The LSU ability to measure the physical composition of urban morphology is also explored and tested. In this paper we use Landsat 7 ETM+ multispectral images and SPOT 5 HRVIR, evaluating at the same time the land use/cover signatures evolution and the effect of spatial resolution differences on the same signatures measurements.

Key words: Remote sensing, Linear Spectral Unmixing, urban land use.

Résumé - ClASSIFICATION DE L'UTILISATION DU SOL URBAIN, PAR ANALYSE LINÉAIRE DE MÉLANGE SPECTRAL D'IMAGES DE SATELLITE. Les milieux urbains étant hétérogènes, on doit les simplifier, en combinant leurs éléments de base d'usage et d'occupation du sol, afin de permettre leur étude quantitative. Le modèle VIS proposé par Ridd en 1995 est un modèle conceptuel qui permet de simplifier les milieux urbains en combinant trois éléments de base: la végétation (V), l'étendue imperméable (I) et le sol nu (S). La plupart des utilisations urbaines peuvent être interprétées à partir des combinaisons entre ces trois éléments. L'analyse de la composition du VIS permet de vérifier que la plupart des caractéristiques urbaines présentent une combinaison particulière de ces trois facteurs, laquelle serait de détermination difficile en utilisant les méthodes de classification pixel par pixel des images satellitaires. On a examiné les caractéristiques des classes d'utilisation et d'occupation du sol urbain dans la Grande Aire Métropolitaine de Lisbonne (GAML), en utilisant des techniques de classification basées sur le sub-pixel, en particulier 1'Analyse de Mélange Spectral (AME), en mettant au point un modèle conceptuel caractérisant les types d'occupation, qu'on a utilisé et testé en l'appliquant à l'AME, afin de mesurer la nature physique de la morphologie urbaine à partir d'images multispectrales Landsat 7 ETM + et SPOT 5 HRVIR. On a aussi évalué l'influence que les différences de résolution spatiale peuvent avoir sur les résultats obtenus.

Mots-clés: Télédétection, Analyse de Mélange Spectral, utilisation du sol urbain.

\section{INTRODUÇÃO}

Um tema recorrente em estudos de detecção remota em meio urbano está relacionado com a obtenção de indicadores sumários para os usos que o compõem. Esta temática tem sido tradicionalmente limitada devido à heterogeneidade espectral dos elementos urbanos em relação à definição espacial dos sensores orbitais utilizados (Forster, 1985). Isto é particularmente verdadeiro no contexto de imagens multiespectrais com definição geométrica média, como é o caso das fornecidas pelo satélite actual Landsat $(30 \mathrm{x} 30 \mathrm{~m})$. Devido a esta heterogeneidade espectral torna-se necessário lidar com uma mistura complexa de respostas espectrais. A existência de misturas espectrais nos pixels (pixels "não puros" ou mixels), das imagens geralmente disponíveis em detecção remota, dificulta a identificação de 
classes de uso do solo urbanas através de técnicas analíticas pixel-a-pixel e constitui o problema mais significativo da detecção remota em meio urbano (Card, 1993). Esta identificação torna-se ainda mais difícil quando o continuum urbano não pode ser prontamente dividido em classes discretas, como é requerido por estas técnicas.

Nos últimos dez anos, tem prevalecido a tendência para adoptar uma forma "flexível" de descrever o carácter espacial da variação do uso do solo em termos de superfícies contínuas. Na abordagem "flexível”, as proporções dos diferentes componentes do uso do solo são estimadas para cada pixel da imagem, representando cada tipo de uso como uma superfície contínua da variação. A classificação fuzzy e a Análise da Mistura Espectral (AME) são duas técnicas utilizadas para fornecer uma análise "flexível" dos mixels. As duas aproximações têm as suas vantagens e desvantagens. Por exemplo, a abordagem fuzzy não restringe o grau de valores de pertença atribuídos a um pixel em diferentes imagens fuzzy de forma a somar uma unidade, embora tenha em consideração as interacções não lineares entre os diferentes tipos de usos do solo que são negligenciadas pela AME. No pólo oposto, os modelos AME representam uma aproximação determinista que transforma os valores da imagem em variáveis físicas, tornando assim mais fácil comandar a análise e inferir resultados do que no caso dos modelos fuzzy que são baseados em métodos estatísticos.

$\mathrm{O}$ trabalho aqui apresentado baseia-se na abordagem AME. Nesta assumese que a paisagem é formada a partir de variações contínuas das proporções de tipos idealizados de usos do solo com espectros puros, chamados membros finais. Os membros finais são reconhecidos na imagem como sendo abstracções de materiais com propriedades uniformes que compõem o uso do solo. Num ambiente urbano, estes podem incluir superfícies impermeabilizadas, espaços verdes, espelhos de água e solo a descoberto. A AME linear corresponde ao processo de resolução das fracções dos membros finais, supondo que o espectro medido para cada pixel representa uma combinação linear de espectros do membro final, que corresponde à mistura física de alguns componentes da superfície, ponderada pela área total. Com a AME, as (fracções de) áreas dos membros finais são quantificadas ao nível do sub-pixel, permitindo a inferência das características morfológicas da paisagem urbana em termos da composição dos membros finais. A finalidade deste artigo é explorar e testar a aplicabilidade da AME para medir a composição física da morfologia urbana, através de imagens multiespectrais Landsat ETM+ e SPOT 5, avaliando ao mesmo tempo o efeito que as diferenças de resolução espacial podem ter nos resultados obtidos.

\section{AS CLASSIFICAÇÕES DE USO E OCUPAÇÃO DO SOLO E O MODELO VIS}

A melhoria das classificações de uso e ocupação do solo tem sido um tema recorrente na literatura relacionada com a detecção remota. Tem sido desenvolvida uma grande variedade de abordagens cujo objectivo é diferenciar as 
classes de uso e ocupação do solo através de dados multiespectrais. Richardson e Wiegand (1977) desenvolveram um tipo de índice de vegetação perpendicular, onde o relacionamento positivo entre as reflectâncias na região do vermelho e infravermelho próximo se traduz numa recta, muitas vezes designada de linha dos solos e que nos ambientes urbanos seria mais correctamente designada de linha da não vegetação. $O$ aumento da distância perpendicular a esta linha representa um aumento na cobertura de vegetação. Kauth e Thomas (1976) desenvolveram transformações ortogonais de imagens em "bruto", que resultam em índices de brilho e de vegetação.

Os índices de vegetação (IV) derivados de imagens de satélite, os descritores do solo ou os rácios de bandas são utilizados várias vezes, como fonte de informação, para aceder a certos componentes da superfície (Jensen, 2000). O facto do tipo de solo poder influenciar os IV, especialmente em áreas com baixa taxa de cobertura ou, por outro lado, a vegetação natural poder esconder ou alterar significativamente a resposta espectral do solo, torna difícil o desenvolvimento separado de índices de Solo e Vegetação. Parece mais natural e promissor utilizar técnicas de decomposição espectral dedicada, como as de separabilidade espectral, para derivar este tipo de informação em apenas um passo.

Um dos maiores avanços nas análises de uso e ocupação do solo foi conseguido com o modelo VIS de Ridd (1995), proposto para estudar a morfologia urbana da área metropolitana de Salt Lake City, examinando imagens TM e fotografias aéreas. O modelo conceptual VIS representa a composição de um ambiente urbano como uma combinação linear de três tipos de ocupação: vegetação verde (V - Vegetation), superfícies impermeabilizadas (I - Impervious surfaces) e solo (S - Soil). Tal como os solos podem ser descritos em termos das suas proporções de limo, areia, e argila, utilizando um diagrama triangular tradicional (Varennes, 2003), assim também várias subdivisões de áreas urbanas podem ser descritas em termos de proporção da Vegetação, Solo e Superfície Impermeável. Este modelo fornece as orientações para decompor as paisagens urbanas e ligar esses componentes às características espectrais dos dados remotos.

A figura 1 corresponde a uma generalização esquemática dos ambientes periurbanos dentro do modelo VIS. São utilizadas as designações tradicionais de usos do solo apenas por conveniência, tendo em vista a transmissão do conceito através da identificação de partes familiares da cidade. O objectivo do VIS não é identificar áreas (classes) de uso solo, mas sim identificar e caracterizar as variações de padrões de uso na cidade. Qualquer correlação com os usos do solo é interessante, mas não representa um objectivo conceptual do VIS. Ao longo do eixo V-I (fig. 1) assenta uma sequência residencial típica de muitas cidades do mundo desenvolvido. Por regra, as áreas industriais e comerciais tradicionais colocam-se próximo do eixo I-S (fig. 1), mas os parques tecnológicos e os espaços industriais modernos desviam-se um pouco para a esquerda, dependendo da quantidade de vegetação presente (quase sempre relva e por vezes árvores). $\mathrm{O}$ facto de determinadas categorias de uso, como os parques ou as escolas, poderem ser encontradas em várias partes do diagrama triangular 
demonstra a necessidade de uma classificação de ocupação do solo baseada em composições. Isto porque é a ocupação do solo e não o uso do solo que pode ser obtida por detecção remota. O uso do solo apenas pode ser inferido pelo interpretador, pois não gera uma assinatura espectral.

O eixo S-V (fig. 1) representa áreas que ainda não foram urbanizadas, ou espaços urbanos em modificação. Por regra, existe pouca área impermeabilizada nas áreas não urbanas ou pré-urbanas. No canto direito, o Solo a descoberto pode representar terrenos no limite ou no interior da cidade terraplanados para urbanização. As áreas de pousio ou de vegetação escassa possuem, como é óbvio, pouca vegetação, enquanto que as culturas por sulcos representam tipicamente uma mistura de Vegetação e Solo e portanto a sua assinatura VIS migra para a esquerda entre a cultura e a colheita. As florestas, dependendo da dimensão e densidade das copas e da vegetação rasteira, apresentam quantidades variáveis de Solo a descoberto, variando também a sua própria composição ao longo do ano. No canto verde encontram-se as culturas extensivas e contínuas, como as pastagens, e os elementos urbanos como os campos de golfe, parques, pátios escolares e outros espaços verdes, privados ou públicos. O espaço verde privado é normalmente ignorado nas classificações de uso do solo; ele pode, no entanto, atingir maior percentagem do meio urbano que o espaço verde público.

O modelo VIS constitui uma ligação intuitivamente apelativa ao problema da mistura espectral, porque a contribuição espectral dos seus três componentes principais pode ser resolvida ao nível do sub-pixel usando a técnica de AME. Este modelo foi originalmente aplicado às cidades americanas. Fora dos Estados Unidos, o VIS também foi testado na Austrália, onde foi utilizada uma classificação hierárquica não supervisionada para classificar - numa base pixel-a-pixel - em quatro classes (água, floresta, espaços livres e urbano) as imagens TM correspondentes ao Sudeste de Queensland (Ward et al., 2000). No final foi alcançada uma exactidão global ajustada de $83 \%$. Para a mesma área, Phinn et al. (2002) compararam a tradicional classificação de imagens (TM), a interpretação de fotografias aéreas e análise linear de mistura espectral (TM) e chegaram à conclusão que as imagens fraccionárias do VIS garantiam uma melhor classificação que os métodos pixel-a-pixel e a agregação de classes obtidas por foto-interpretação. Na Tailândia foi utilizado um classificador não supervisionado (ISODATA) para classificar dados TM da Área Metropolitana de Banguecoque em sete classes (Madhavan et al., 2001). O modelo VIS provou ser útil para a classificação, embora devido à complexidade da área em estudo a exactidão não tenha sida tão elevada como era inicialmente esperado. Rashed et al. (2001) conduziram uma análise de mistura espectral em imagens multiespectrais IRS-1C com o intuito de descrever a anatomia da região do Grande Cairo (Egipto). Esta análise foi baseada em quatro membros finais (Vegetação, superfície Impermeável, Solo e Sombra) cujas imagens de probabilidade foram classificadas com recurso a uma árvore de decisão, tendo a exactidão da classificação sido melhor que as obtidas pelos classificadores de mínima distância e máxima verosimilhança. Estes resultados 
mostram que o modelo é robusto fora dos Estados Unidos da América, embora por vezes possa requerer um componente adicional (por exemplo uma máscara binária água/não água) para conseguir uma caracterização exacta da morfologia das cidades não americanas. Em 2003, Wu e Murray recorreram à AME para analisar a distribuição de superfície impermeabilizada na Área Metropolitana de Columbus (Ohio - EUA) e descobriram que esta pode ser estimada através de um modelo de regressão linear de fracções de membros finais correspondentes a baixo e alto albedo.

Mais recentemente, Lu e Weng (2004) encetaram um estudo para analisar a mistura espectral da paisagem urbana da cidade de Indianápolis, utilizando imagens Landsat ETM+. Neste estudo foram seleccionados quatro tipos de membros finais: Sombra, Vegetação verde, Superfície Impermeabilizada e Solos. Para encontrar as melhores imagens fraccionárias foram testadas quatro combinações destes membros finais: 1) quatro membros finais com sombra, vegetação verde, superfície impermeabilizada e solos; 2) três membros finais com sombra, vegetação verde e superfície impermeabilizada; 3) três membros finais com sombra, vegetação verde e solos (secos) e 4) três membros finais com sombra, vegetação verde e solos (húmidos). Os resultados indicaram que a combinação 2) produzia bons resultados em áreas urbanas mas relativamente pobres em áreas agrícolas, especialmente em solos descobertos e pastagens. Em contraste, a combinação 4) não provou a sua utilidade em áreas urbanas mas resultou bastante bem em áreas agrícolas. A combinação 1) apresentou maus resultados em ambos os domínios, com particular incidência nas áreas urbanas residenciais. A melhor solução encontrada foi a combinação 2) que consegue relativamente bons resultados, tanto em meio urbano como não urbano (fig. 2).

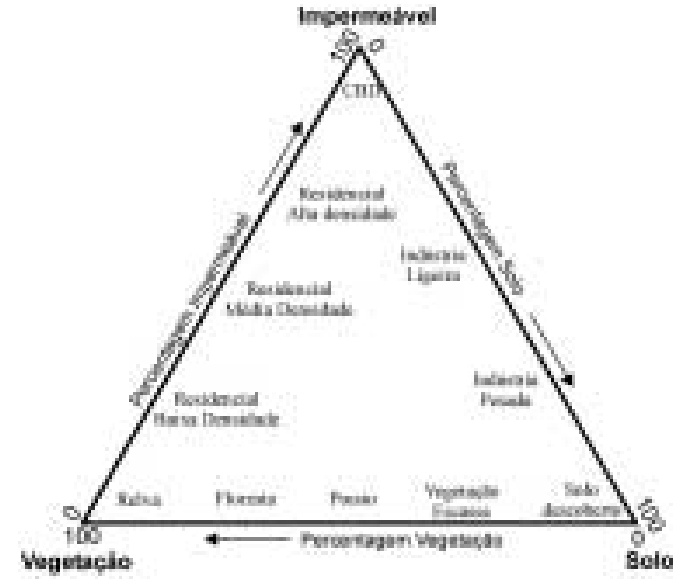

Fig. 1 - Representação de alguns usos urbanos e sub-urbanos no modelo VIS.

Fig. 1 - Representation of urban and suburban land uses through the VIS model.

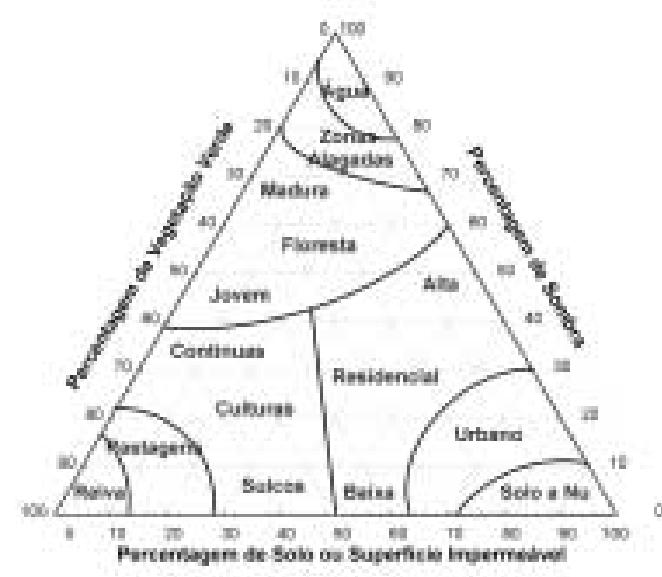

Fig. 2 - Modelo da paisagem urbana. Adaptado de Lu e Weng (2004).

Fig. 2 - Urban landscape model. Adapted from Lu and Weng (2004). 


\section{ANÁLISE DE USO E OCUPAÇÃO DO SOLO}

Os dados utilizados para este trabalho foram imagens Landsat (7) ETM+ de 2000 e SPOT (5) HRVIR de 2004, ambas obtidas com céu limpo. No caso do Landsat, a utilização da banda 6 foi declinada em virtude da sua definição espacial grosseira. A área geográfica de análise corresponde à Grande Área Metropolitana de Lisboa, constituída por dezoito municípios.

Antes de se dar início à análise os dados foram convertidos adiometricamente para a reflectância ao nível do sensor, através de um método de correcção baseado nas imagens (Markham e Barker, 1987). Depois, todas as imagens foram georreferenciadas relativamente às cartas topográficas, à escala 1:25000 do Instituto Geográfico do Exército (IgeoE), produzidas segundo o sistema de projecção Hayford-Gauss (transversa de Mercator - datum Lisboa). De referir que, apesar da imagem SPOT já se encontrar geometricamente corrigida, a sua exactidão geométrica não era suficiente para permitir a comparação entre dados multisensor e o sistema de projecção era diferente do utilizado (Universal Transverse Mercator - UTM), pelo que se justificou a sua nova rectificação. Depois de georreferenciadas, as imagens foram reamostradas para uma dimensão de pixel de $10 \mathrm{~m}$ (por ser a maior resolução - SPOT - e por a divisão do pixel Landsat $(30 \mathrm{~m})$ por este valor resultar num número inteiro) através do método do vizinho mais próximo. Para ambas as imagens conseguiu-se obter um erro médio quadrático inferior a 0,5 do pixel original.

\section{Definição dos componentes do solo e imagens de percentagem resultantes}

Este estudo baseia-se no modelo VIS, pelo que foram seleccionados três componentes do solo como sendo a estrutura básica dos usos urbanos. Assim, foram escolhidas as percentagens de Vegetação (árvores, arbustos, relva), Solo Impermeável (telhados, metal, betão, asfalto) e Solo a nu. Este último engloba também a vegetação seca porque estes dois tipos de ocupação têm características similares de reflectância espectral e, obviamente, de níveis radiométricos nas imagens de satélite (Hoffer, 1978).

A aplicação bem sucedida da AME confia na exactidão da selecção do membro final. Se os membros finais estiverem incorrectos no sentido físico, então as percentagens também estão incorrectas e os resultados da AME não fazem sentido. A selecção dos membros finais pode ser feita de cinco maneiras: 1) de uma biblioteca espectral, ou reflectâncias medidas no solo; 2) da própria imagem (Settle e Drake, 1993) ou dos vectores próprios de ordem superior de uma classificação em componentes principais (Boardman, 1993); 3) de pixels espectralmente puros, identificados através do índice de pureza espectral; 4) da selecção manual dos membros finais (Bateson e Curtiss, 1996), correspondente a uma técnica de visualização multidimensional que permite explorar interactivamente o espaço de mistura à procura de espectros que possam 
ser designados de membros finais e 5) da combinação dos métodos utilizados para obter os membros finais de referência e da imagem. Esta abordagem envolve um alinhamento espectral entre os dois tipos de membros finais (referência e imagem) e uma calibração que os relacione (Roberts et al., 1993). Na maioria de aplicações da AME são utilizados os membros finais da imagem porque estes podem ser facilmente obtidos e representam espectros medidos na mesma escala que a imagem (Roberts et al., 1998). Os membros finais são tidos como os extremos dos triângulos no diagrama de dispersão de uma imagem. Deste modo, podem ser identificados através do diagrama de dispersão de duas bandas espectrais. $\mathrm{Na}$ ausência de membros finais de referência para a área de estudo, optou-se pela utilização dos membros finais da imagem na fase da AME.

O componente Vegetação foi facilmente identificado em campos de golfe (por ex. Aroeira), o componente Impermeável em áreas residenciais (especialmente de alta densidade) e o componente Solo nos sopés das serras (Arrábida e Sintra) ou dentro das áreas agrícolas (pousio). A análise das imagens correspondentes aos componentes VIS das imagens Landsat e SPOT mostra que, como seria de esperar, as áreas com maior percentagem de vegetação são os campos de golfe e os parques. As áreas com grande percentagem de impermeabilização correspondem às zonas residenciais, industriais e comerciais. Por fim, as áreas onde predomina o componente Solo identificam-se com o solo a descoberto, normalmente inserido em áreas agrícolas (pousio, terrenos abandonados) ou urbanas (terreno em reconversão).

Como a selecção dos membros finais é fundamental para uma boa classificação tentou-se ainda melhorar o resultado final, tornando as áreas teste mais puras em termos espectrais. Para atingir este objectivo utilizou-se um método e outro não paramétrico (McKay e Campbell, 1982). Ambos os métodos de purificação forneceram resultados iguais, não melhorando o resultado do RMS, facto que demonstra a boa qualidade das áreas de treino iniciais.

\section{Análise de Uso e Ocupação do Solo}

Para demonstrar as potencialidades da utilização dos componentes VIS na análise do uso e ocupação do solo urbano foram seleccionadas diversas classes de uso e ocupação do solo sobre a imagem. Estas classes correspondem às utilizadas no projecto CartusAML (Tenedório et al., 1999), cujo resultado final se traduziu numa carta de uso e ocupação do solo para a Área Metropolitana de Lisboa (igual à GAML, mais o concelho da Azambuja), obtida através da foto-interpretação de fotografia aérea à escala 1:30 000 e correspondente ao ano de 1991. Depois de identificadas as classes, extraíram-se os seus componentes e representaram-se num diagrama triangular. A figura 3 mostra a composição VIS das classes urbanas num gráfico triangular, quer para uma resolução espacial de $30 \mathrm{~m}$ (Landsat), quer de $10 \mathrm{~m}$ (SPOT). Muito embora estas classes urbanas não sejam fáceis de distinguir visualmente sobre 
uma imagem, sem anterior informação sobre a área de estudo, a tabela associada à figura 3 prova que cada elemento urbano possui uma assinatura VIS única. Esta assinatura é tão característica que é possível delinear territórios com base nessa composição de VIS e atribuir esses territórios a classes de usos urbanos. É de notar que os estudos anteriores levados a efeito neste âmbito têm-se cingido a classes muito gerais, para obviar o problema das excepções (valores extremos), nunca nenhum se aproximando do valor de dezanove classes aqui apresentado.

Observando o diagrama triangular (fig. 3a) referente à imagem Landsat verifica-se que existem sete classes com maior percentagem de Solo Impermeável $(4,8,13,14,15,16$ e 19), sete com Vegetação $(2,3,7,9,10$, 12 e 17) e cinco com Solo (1, 5, 6, 11 e 18). No primeiro caso, o resultado corresponde quase exclusivamente às classes urbanas, industriais e comerciais, aparecendo como excepção as praias e os areeiros, cujas reflectâncias espectrais são muito semelhantes à do betão, gerando normalmente erros de classificação. Nas áreas verdes os elementos anómalos são as áreas de água, ou alagadas, o que está directamente relacionado com o facto de não se ter utilizado uma máscara para a água; como a grande maioria destas áreas são sapais ou lagoas (águas paradas com muita vegetação) a sua resposta espectral aproxima-se da Vegetação. Quanto aos grandes equipamentos e áreas militares, o seu aparecimento nesta classe mostra que os grandes equipamentos são basicamente parques naturais e campos de golfe e que os edifícios militares se encontram disseminados entre a vegetação. Por fim, no terceiro caso, temos todas as classes onde existe muito Solo a descoberto, incluindo a habitação unifamiliar onde cada habitação é normalmente acompanhada de uma pequena parcela de terreno.

Quanto aos resultados observados para as mesmas classes (fig. 3b), mas com uma resolução espacial de $10 \mathrm{~m}$ (SPOT), verificou-se que existiam nove classes com maior percentagem de solo Impermeável $(3,4,7,8,12,13,14,16$ e 19), dez com maior percentagem de Vegetação $(1,2,5,6,9,10,11,15,17$ e 18) e nenhuma com maior percentagem de Solo.

Em termos gerais pode dizer-se que a passagem de uma resolução espacial de $30 \mathrm{~m}$ para uma de $10 \mathrm{~m}$ (fig. 4), origina uma rotação, no sentido horário, de alguns usos dentro do gráfico triangular. Numa observação mais cuidada, classe a classe, verifica-se um aumento da percentagem de Impermeabilização nas classes 7, 8, 13, 14 e 19, e principalmente 3,12 e 1; antagonicamente, nas classes 5, 11 e 15, observa-se uma diminuição da percentagem de Impermeabilização. As restantes classes, nomeadamente a 1, 2, 6, 9, 10 e 18, são caracterizadas pelo aumento da percentagem de Vegetação, com excepção da classe 4, que é a única que se mantém estável.

Estes resultados indicam que a questão da dimensão do pixel é bastante importante na classificação de imagens de satélite em geral e na análise dos componentes VIS em particular. Efectivamente, as bandas ETM+, com os seus $30 \mathrm{~m}$ de resolução, tornam possível identificar vários materiais de cobertura do 



Fig. 3 - Componentes VIS das classes de uso do solo (Landsat e SPOT).

Fig. 3 - Land use classes VIS components (Landsat and SPOT).

\begin{tabular}{|c|c|c|c|c|c|c|c|}
\hline \multirow{2}{*}{$\begin{array}{l}\text { Identi- } \\
\text { ficação }\end{array}$} & \multirow{2}{*}{ Designação } & \multicolumn{3}{|c|}{ Landsat (30m) - fig.3a } & \multicolumn{3}{|c|}{ SPOT (10m) - fig. 3b } \\
\hline & & $\mathrm{V}(\%)$ & I (\%) & S (\%) & $\mathrm{V}(\%)$ & I (\%) & S (\%) \\
\hline A & Área agrícola & 36 & 11 & 52 & 50 & 15 & 35 \\
\hline Fm & Área florestal e matas & 60 & 08 & 32 & 69 & 05 & 26 \\
\hline Am & Planos de água, marinhas e salinas & 63 & 12 & 25 & 41 & 48 & 11 \\
\hline $\mathrm{Ca}$ & Núcleo urbano consolidado & 25 & 46 & 29 & 25 & 46 & 29 \\
\hline $\mathrm{Hu}$ & Área de habitação unifamiliar & 33 & 34 & 34 & 37 & 32 & 32 \\
\hline Iv & Terrenos incultos e vazios & 42 & 17 & 42 & 58 & 18 & 24 \\
\hline $\mathrm{Ge}$ & Área de grandes equipamentos & 40 & 38 & 22 & 34 & 49 & 17 \\
\hline $\mathrm{Hp}$ & Área de habitação plurifamiliar & 26 & 58 & 16 & 20 & 63 & 17 \\
\hline Im & Área de instalações militares e fortificações & 44 & 25 & 31 & 54 & 24 & 21 \\
\hline M & Mato & 57 & 09 & 34 & 72 & 08 & 19 \\
\hline $\mathrm{L}$ & Loteamento & 32 & 32 & 36 & 39 & 29 & 33 \\
\hline $\mathrm{Sa}$ & Sapal e outras áreas alagadas & 82 & 11 & 07 & 32 & 65 & 03 \\
\hline $\mathrm{Pc}$ & Praia, arribas e formações vegetais costeiras & 40 & 40 & 20 & 26 & 53 & 21 \\
\hline $\mathrm{Pi}$ & Área portuária, industrial e de armazenagem & 32 & 48 & 20 & 22 & 60 & 17 \\
\hline Ap & Areeiro/Pedreira & 27 & 40 & 33 & 36 & 31 & 33 \\
\hline Gc & Grandes superfícies comerciais & 44 & 52 & 04 & 09 & 90 & 02 \\
\hline $\mathrm{Av}$ & Área verde urbana & 66 & 19 & 15 & 67 & 24 & 09 \\
\hline AA & Área agro-florestal & 34 & 02 & 64 & 55 & 01 & 44 \\
\hline $\mathrm{Mu}$ & Área multifuncional metropolitana & 24 & 67 & 10 & 18 & 69 & 13 \\
\hline
\end{tabular}

solo com assinaturas espectrais características (Wheeler, 1985). Num estudo de uso e ocupação do solo na área de Toronto, com utilização de imagens TM, Gong e Howarth (1990) conseguiram distinguir doze tipos de cobertura (seis de Vegetação, três de Impermeável, duas de Solos e a Água). Uma abordagem similar, levada a efeito na cidade de Salt Lake (Wheeler, 1985), concluiu que um quinto dos pixels era claramente classificado dentro de um dos componentes 
VIS, sendo os restantes mixels representantes de diferentes proporções dos três componentes. Chung (1989) estudou a mesma área, mas recorreu a imagens SPOT XS (20m), tendo concluído que $38 \%$ dos 776 pixels de amostra eram puros, para uma classificação em sete classes (árvores/arbustos, relva, alcatrão, betão, telhados, solo e água). Combinando estas classes em apenas quatro (VIS mais água), a percentagem de pixels puros subia para $67 \%$. No entanto, os restantes $33 \%$, condicionados pela disponibilidade de apenas três bandas espectrais, continuavam a apresentar uma considerável confusão entre Impermeável e Solo. Mais recentemente, num estudo que recorreu a imagens IKONOS para quantificar as combinações de características espaciais e espectrais das reflectâncias urbanas de catorze áreas urbanas distribuídas por todo o mundo, demonstrou-se que a dimensão dos elementos urbanos variava normalmente entre os 10 e os $20 \mathrm{~m}$ (Small, 2003). Isto explica o facto das áreas urbanas serem caracterizadas pela heterogeneidade quando são observadas por sensores de média resolução (20-30m), sugerindo ao mesmo tempo que uma significativa fracção dos pixels das bandas multiespectrais IKONOS $(4 \mathrm{~m})$ sejam heterogéneos. Assim, só uma conclusão se pode tirar: ainda vão ser precisos mais estudos para determinar a combinação óptima de resolução espacial e resolução espectral para estudos urbanos. A certeza consiste em haver um limite para a diminuição da dimensão do pixel e o problema dos mixels (embora em menor intensidade) que continuará presente.

\section{Estrutura do Uso e Ocupação do Solo na GAML}

A estrutura do uso e ocupação do solo na Área Metropolitana de Lisboa revela uma repartição muito desigual considerando as grandes classes de uso do solo (fig. 5): Áreas Edificadas, Áreas Agrícolas e Áreas Florestais (incluindo os meios "naturais e semi-naturais") e uma distribuição assimétrica entre a margem Norte e a margem Sul do Tejo. As três classes de uso do solo supracitadas foram obtidas por agrupamento de um conjunto de dezanove subclasses. As Áreas Edificadas resultam da agregação de onze classes de uso: Área edificada consolidada antiga, Área multifuncional metropolitana, Área residencial de edifícios plurifamiliares, Área residencial de edifícios unifamiliares, Área de grandes equipamentos e infra-estruturas, Área portuária, industrial ou de armazenagem, Grandes superfícies comerciais, Área de extracção de inertes, Área militar, Loteamentos e Área verde urbana. A classe de Áreas Agrícolas é composta por Área agrícola e Área de actividade agrícola abandonada, incultos e vazios urbanos. Finalmente, a classe de Áreas Florestais reúne Área de montado, Área de mato, Área florestal e matas, e ainda os meios "naturais" ou "semi-naturais", praias, arribas e formações vegetais costeiras, sapal e outras áreas alagadas e Planos de água, marinhas e salinas.

Atendendo ao peso percentual que cada classe de uso ocupa no total da área de cada concelho, poder-se-ão criar agrupamentos de concelhos com uma distribuição semelhante de repartição percentual de usos do solo. Face ao exposto 




Fig. 4 - Variação da composição de VIS em cada classe de uso devido à variação da resolução espacial.

Fig. 4 - Variations in VIS components due to spatial resolution change.

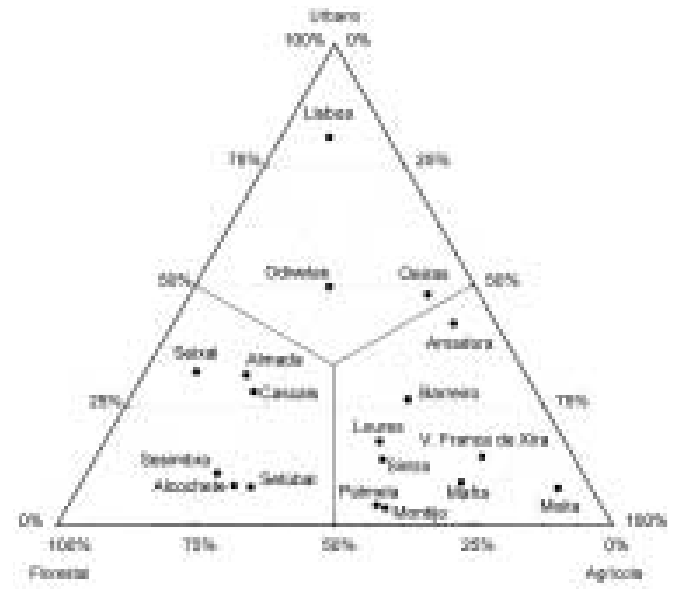

Fig. 5 - Concelhos da GAML segundo três classes de uso do solo (obtidos por aglomeração das classes obtidas por foto-interpretação).

Fig. 5 - Triangular diagram of GAML municipalities according to three main land use classes (obtained through generalization of the land use classes obtained by photo-interpretation).

é possível estabelecer três conjuntos de concelhos com características distintas: a) Amadora, Cascais, Odivelas, Oeiras, Almada e Seixal, com predomínio das áreas edificadas (30\%-50\%) e áreas florestais (37\%-65\%); b) Sesimbra, Setúbal, Sintra, Loures e Barreiro, com predomínio das áreas agrícolas (20\%-42\%); c) Mafra, Vila Franca de Xira, Palmela, Montijo e Moita, onde prevalecem as áreas agrícolas (59\%-82\%). O concelho de Lisboa, apresenta uma estrutura singular já que tem cerca de $80 \%$ de áreas edificadas, contando apenas com aproximadamente $11 \%$ de áreas florestais e $8 \%$ de áreas agrícolas.

Considerando a estrutura de uso e a ocupação do solo representada nas figuras 5 e 6 , podem-se formar, ainda que a título exploratório, os seguintes grupos:

- o centro da metrópole ancorado em Lisboa por assumir, de forma singular, valores de área edificada substancialmente superiores aos restantes concelhos;

- a periferia de franja urbana fragmentada com três subgrupos: a) Sintra, Loures e Barreiro com uma estrutura de uso preponderantemente agrícola (aproximadamente 50\%) mas com peso significativo de áreas edificadas; b) Odivelas, Oeiras e Amadora com dominância de áreas edificadas (cerca de 50\%) mas já de transição para a dominância de territórios agrícolas abandonados e vazios urbanos; c) Seixal, Almada e Cascais 
preponderantemente florestal (entre 50 a 60\%) mas com área edificada assinalável (aproximadamente 30\%);

- a periferia florestal, integrando meios "naturais" e "semi-naturais", constituída por Alcochete com predomínio de Área de montado, Sesimbra e Setúbal integrando a área pertencente ao Parque Natural da Serra de Arrábida, com valores compreendidos entre 60 e 70\%;

- a periferia agrícola composta por Mafra, Moita, Vila Franca de Xira (integrando a lezíria do Tejo), Palmela e Montijo (estes dois últimos numa posição de transição para o Alentejo assumindo valores significativos de Área florestal.

Adaptando os componentes VIS ao nível do concelho (valor médio por unidade territorial) também se obtêm resultados bastante interessantes. A observação com base nas imagens Landsat (fig. 6a) conduz a uma descriminação de quatro grandes grupos de concelhos. $\mathrm{O}$ primeiro, correspondente a áreas fortemente Impermeabilizadas respeita os concelhos de Lisboa e Amadora. O segundo, onde predomina o Solo, é constituído pelos concelhos agrícolas da GAML: Moita, Palmela, Montijo e Alcochete. Por fim, o grupo onde prevalece a Vegetação pode ser subdividido em dois subgrupos; um, que já apresenta um forte componente de Impermeabilização e que é constituído pelos concelhos de Oeiras, Odivelas, Barreiro, Seixal, Almada e Cascais; outro, onde a Impermeabilização ainda não se faz sentir (percentualmente) tão fortemente e que agrupa os concelhos de Loures, Sintra, Sesimbra, Setúbal, Mafra e Vila Franca de Xira. Ao passar para as imagens SPOT (fig. 6b) verifica-se que Oeiras passa a incorporar o grupo dos mais impermeabilizados, que a separação entre os dois subgrupos dos mais vegetalizados torna-se mais ténue (não esquecer que para além de uma diferença de resolução, há uma diferença de datas entre as duas imagens) e que o fenómeno verificado aquando da análise das classes de uso perda de importância relativa por parte dos solos - também aqui se faz sentir com bastante incidência, implicando que, à excepção de Palmela, todos os concelhos que se encontravam no grupo de predominância dos Solos passem a estar incluídos no de predominância da Vegetação.

\section{CONCLUSÃO}

As paisagens urbanas são complexas e muitas vezes difíceis de classificar. Este estudo demonstra que a AME é uma abordagem válida para caracterizar os padrões de uso e ocupação do solo em áreas urbanas. Uma vez que estes usos tendem a ser compostos por misturas espectrais heterogéneas, mesmo quando observados a grandes resoluções espaciais, a utilização da AME para melhorar as classificações de uso e ocupação do solo é especialmente válida. Isto é particularmente verdadeiro se considerarmos que o aumento da resolução espacial produz um aumento do custo (de aquisição e de processamento) e 

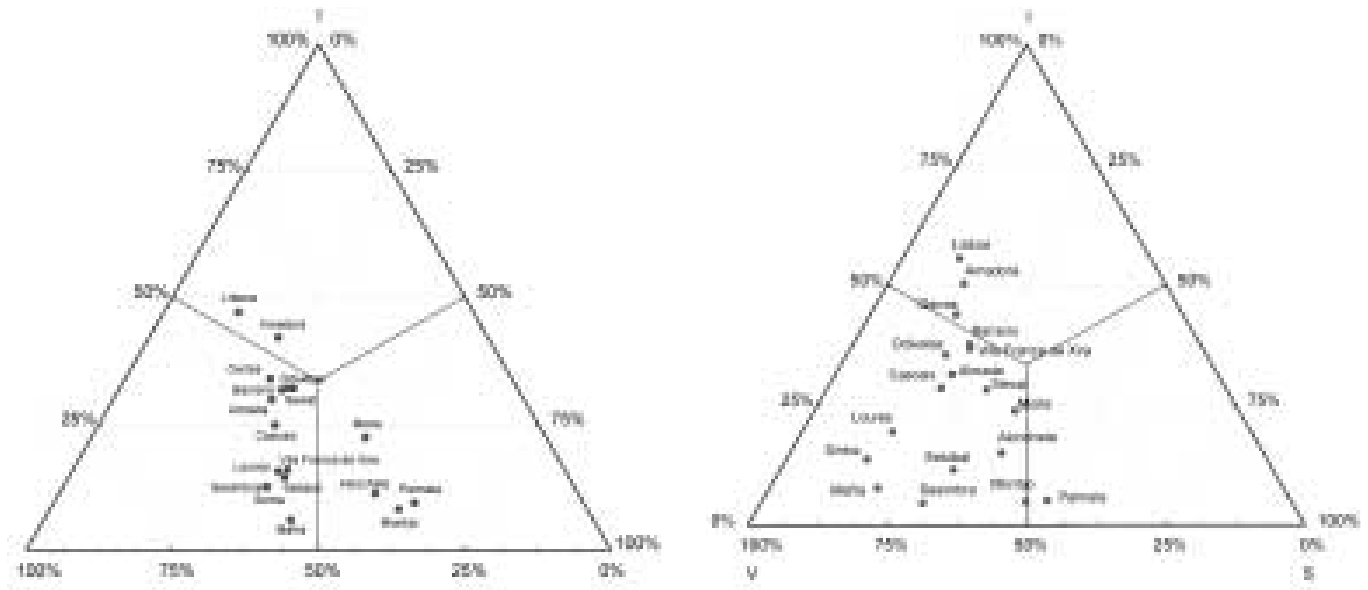

Fig. 6 - Composição VIS dos concelhos da GAML (Landsat e SPOT).

Fig. 6-GAML municipalities VIS composition (Landsat e SPOT).

da variação espectral dos tipos de uso e ocupação do solo, conduzindo, paradoxalmente, a um decréscimo da separabilidade espectral das classes e, consequentemente, a uma redução na exactidão das classificações. Neste estudo verificou-se que através da AME é possível identificar de forma unívoca as classes de uso e ocupação do solo e que esta abordagem também é válida para caracterizar a estrutura de uso e ocupação do solo ao nível concelhio. Ficou também provado que a resolução espacial é importante, mas não fundamental, na medida em que os resultados obtidos pelas imagens Landsat $(30 \mathrm{~m})$ pareceram, face ao conhecimento da área de estudo, bastante mais fáceis de interpretar e/ou justificar, que os obtidos através da análise das imagens SPOT $(10 \mathrm{~m})$.

O modelo VIS provou ser um suporte válido para a AME. As imagens resultantes fornecem informação ao nível do sub-pixel sobre os componentes VIS das áreas urbanas, permitindo a representação simplificada de áreas heterogéneas como combinações de componentes básicos do solo. Deste modo, os estudos urbanos podem ser conduzidos não só numa perspectiva qualitativa, mas também quantitativa. A imagem resultante contém uma quantidade considerável de informação que normalmente não é extraível das imagens de satélite através dos classificadores pixel-a-pixel. Esta abordagem pode ser bastante útil em diversos estudos urbanos relacionados com a população, crescimento urbano e alterações de uso.

Apesar de ter demonstrado a sua utilidade na identificação dos padrões de uso do solo, o modelo VIS apresenta certos constrangimentos práticos relacionados com alguns factores. Em primeiro lugar, este modelo não consegue interpretar usos como água ou zonas húmidas. Em resoluções espaciais médias, o núcleo antigo, a indústria, o solo a descoberto e as áreas residenciais são difíceis de diferenciar. Em segundo lugar, a superfície impermeável do modelo VIS 
não é fácil de ser identificada como um membro final, através da análise de imagens de satélite, porque corresponde a uma complexa mistura de diferentes materiais como asfalto, betão, metal, plástico e argila (telha). Finalmente, o modelo VIS exclui um importante componente dos mixels, que é a sombra. A sombra causada pelos grandes edifícios ou árvores é um factor de grande importância, com influência directa na resposta espectral dos tipos de uso e ocupação do solo.

\section{BIBLIOGRAFIA}

Bateson A, Curtiss B (1996) A method for manual endmember selection and spectral unmixing. Remote Sensing of the Environment, 55: 229-243.

Boardman J W (1993) Automated spectral unmixing of AVIRIS data using convex geometry concepts. Summaries of the Fourth JPL Airborne Geoscience Workshop, JPL Publication 93-26, NASA Jet Propulsion Laboratory, Pasadena, Calif.: 11-14.

Card D H (1993) Examination of a simple surface composition model of the urban environment using remote sensing. PhD dissertation, The Department of Geography - The University of Utah, Salt Lake City, Utah.

Chung J J (1989) SPOT pixel analysis for urban ecosystem study in Salt Lake City, Utah. M.S. Thesis, University of Utah, Salt Lake City.

Forster B C (1985) An examination of some problems and solutions in monitoring urban areas from satellite platforms. International Journal of Remote Sensing, 6 (1): 139-151.

Gong P, Howarth P J (1990) The use of structure information for improving land-cover classification accuracies at the rural-urban fringe. Photogrammetric Engineering and Remote Sensing, 56: $67-73$.

Hoffer R M (1978) Biological and physical considerations in applying computer-aided analysis techniques to remote sensor data. In Swain P H, Davis S M (ed.) Remote sensing: The quantitative approach. McGraw-Hill, Inc., New York: 227-289.

Jensen J R (2000) Remote sensing of the environment: an earth resource perspective. Prentice Hall, Upper Saddle River, NJ.

Kauth R J, Thomas G S (1976) The tasseled cap-a graphic description of the spectral-temporal development of agricultural crops as seen by Landsat. Proceedings of the Symposium on Machine Processing of Remotely Sensed Data: 4041-4051.

Lu D, Weng Q (2004) Spectral Mixture Analysis of the Urban Landscape in Indianapolis City with Landsat ETM+ Imagery. Photogrammetric Engineering \& Remote Sensing, 70 (9): 1053-1062.

Madhavan B B, Kubo S, Kurisaki N, Sivakumar T V L N (2001) Appraising the anatomy and spatial growth of the Bangkok Metropolitan area using a vegetation-impervious-soil model through remote sensing. International Journal of Remote Sensing, 22:789-806.

Markham B L, Barker J L (1987) Thematic mapper bandpass solar exoatmospheric irradiances. International Journal of Remote Sensing, 8: 517-523.

McKay R J, Campbell N A (1982) Variable selection techniques in discriminant analysis II: Allocation. British Journal of Mathematical and Statistical Psychology, 35: 30-41.

Phinn S, Stanford M, Scarth P, Murray A T, Shyy P T (2002) Monitoring the composition of urban environments based on the vegetation-impervious surface-soil (VIS) model by subpixel analysis techniques. International Journal of Remote Sensing, 23: 4131-4153. 
Rashed T, Weeks J R, Gadalla M S, Hill A G (2001) Revealing the anatomy of cities through spectral mixture analysis of multisepctral satellite imagery: a case study of the Greater Cairo region, Egypt. Geocarto International, 16: 5-15.

Richardson A J, Wiegand C L (1977) Distinguishing vegetation from soil background information. Remote Sensing of the Environment, 8: 307-312.

Ridd M K (1995) Exploring a V-I-S (Vegetation-Impervious Surface-Soil) model for urban ecosystem analysis through remote sensing: Comparative anatomy for cities. International Journal of Remote Sensing, 16 (21): 2165-2185.

Roberts D A, Smith M O, Adams J B (1993) Green vegetation, nonphotosynthetic vegetation, and soil in AVIRIS data. Remote Sensing of the Environment, 44: 255-269.

Roberts D A, Batista G T, Pereira J L G, Waller E K, Nelson B W (1998) Change identification using multitemporal spectral mixture analysis: applications in eastern Amazônia. In Lunetta R S, Elvidge C D (ed.) Remote sensing change detection: environmental monitoring methods and applications. Ann Arbor Press, Ann Arbor, Mich.: 137-161.

Settle J J, Drake N A (1993) Linear mixing and the estimation of ground cover proportions. International Journal of Remote Sensing, 14: 1159-1177.

Small C (2003) High spatial resolution spectral mixture analysis of urban reflectance. Remote Sensing of the Environment, 88: 170-186.

Tenedório J A, Ferreira J C, Rocha J, Sousa P, Mota G, Pontes S (1999) Carta de Uso do Solo da Área Metropolitana de Lisboa (CARTUS-AML). Actas do VIII Colóquio Ibérico de Geografia, DGPR-UNL, Lisboa, vol. II: 711-716.

Varennes A (2003) Produtividade dos solos e ambiente. Escolar Editora, Lisboa.

Ward D, Phinn S R, Murray A L (2000) Monitoring growth in rapidly urbanizing areas using remotely sensed data. Professional Geographer, 53: 371-386.

Wheeler D J (1985) Evaluation of thematic mapper data for determining urban land cover. $\mathrm{PhD}$ dissertation, The Department of Geography - The University of Utah, Salt Lake City, Utah.

Wu C, Murray A T (2003) Estimating impervious surface distribution by spectral mixture analysis. Remote Sensing of the Environment, 84: 493-505. 